\title{
Comparative Effectiveness of Chemotherapy in Elderly Patients with Metastatic Colorectal Cancer
}

\author{
Sacha Satram-Hoang • Luen Lee • Shui Yu • \\ Sridhar R. Guduru • Ashokvardhan R. Gunuganti • \\ Carolina Reyes $\cdot$ Edward McKenna
}

Published online: 7 November 2012

(C) The Author(s) 2012. This article is published with open access at Springerlink.com

\begin{abstract}
Purpose Treatment advances have improved outcomes in clinical trials of patients with metastatic colorectal cancer (mCRC). Less is known about these effects for patients in real-world settings. This study evaluated treatment patterns and survival in older, demographically diverse patients with mCRC.

Methods A retrospective cohort analysis was performed for 4,250 patients from January 1, 2000 to December 31, 2007 using linked Surveillance, Epidemiology, and End ResultsMedicare database. Patients were $\geq 66$ years, enrolled in Medicare parts A and B, and received first-line treatment with fluorouracil and leucovorin (5-FU/LV), capecitabine (CAP), 5-FU/LV plus oxaliplatin (FOLFOX), or CAP and oxaliplatin (CAPOX). Cox regression with backward elimination and propensity score-weighted Cox regression estimated relative risk of death. Date of last follow-up was December 2009. Statistical comparisons were made between 5-FU/LV vs. CAP and FOLFOX vs. CAPOX.

Results Compared to 5-FU/LV, patients treated with CAP were older (mean age 78 vs. 76; $P<0.0001$ ) and more likely female ( 61 vs. $54 \% ; P=0.0017$ ), while patients receiving CAPOX and FOLFOX were similar in age (mean age 74 vs. 73; $P=0.0924)$. Complications requiring medical resource utilization following initiation of therapy were significantly
\end{abstract}

Electronic supplementary material The online version of this article (doi:10.1007/s12029-012-9450-x) contains supplementary material, which is available to authorized users.

S. Satram-Hoang $(\bowtie) \cdot$ S. R. Guduru $\cdot$ A. R. Gunuganti

Q.D. Research, Inc,

8789 Auburn Folsom Road C501,

Granite Bay 95746 CA, USA

e-mail: sacha@qdresearch.com

L. Lee $\cdot \mathrm{S} . \mathrm{Yu} \cdot \mathrm{C}$. Reyes $\cdot$ E. McKenna

Genentech, Inc,

South San Francisco, CA, USA higher among patients administered with 5-FU/LV (54\%) vs. CAP $(17 \% ; P<0.0001)$ and FOLFOX $(75 \%)$ vs. CAPOX (57\%; P<0.0001). The multivariate analysis revealed no significant differences in survival between 5 FU/LV and CAP and between FOLFOX and CAPOX.

Conclusions Overall survival was comparable between CAP and 5-FU/LV and between CAPOX and FOLFOX with fewer complications requiring medical resource utilization associated with CAP and CAPOX, thus confirming clinical trial results.

Keywords Metastatic colorectal cancer - Chemotherapy · Elderly patients $\cdot$ Survival $\cdot$ Treatment-related complications

\section{Introduction}

Colorectal cancer (CRC) is a disease of the elderly with a median age at diagnosis of 70 years and median age at death of 75 years [1]. It is the third most frequently diagnosed cancer as well as the third leading cause of cancer mortality in men and women in the USA [2]. Approximately $20 \%$ of patients are diagnosed with metastatic disease with 5-year survival rates of $11.7 \%$ [1].

For the past 50 years, the mainstay of systemic treatment for advanced or metastatic CRC (mCRC) has been fluoropyrimidines (FP) administered as monotherapy or in combination with leucovorin (LV) or newer agents such as irinotecan (IFL) and oxaliplatin [3,4]. Clinical trials and meta-analyses demonstrate that 5-fluorouracil (5-FU) and LV (5-FU/LV) improve response rates (RRs) and survival among patients with $\mathrm{mCRC}[3,5]$. Randomized controlled trials have also established the efficacy of 5-FU/LV plus oxaliplatin (FOLFOX) with significant improvements in RR and progression-free survival (PFS) when administered as first-line therapy for patients with advanced CRC [6]. 
Capecitabine (CAP) is an oral fluoropyrimidine that is converted to 5-FU. Two randomized, non-blinded phase 3 trials compared single-agent $\mathrm{CAP}$ with 5 -FU/LV as first-line therapy of patients with $\mathrm{mCRC}$ and established that CAP was at least as active as 5-FU/LV in achieving an objective tumor RR, [7, 8] and PFS and overall survival (OS) were equivalent between treatment arms in a prospective pooled analysis of two similarly designed phase 3 trials [9]. Capecitabine and oxaliplatin (CAPOX) have demonstrated clinical activity in multiple clinical trials as first-line treatment for patients with mCRC [10-17] and provide comparable clinical outcomes to FOLFOX $[12,18]$ and infusional 5-FU/ oxaliplatin [19].

Historically, elderly patients have been underrepresented in clinical trials with only one quarter to one third of potentially eligible older patients enrolled in cancer clinical trials [20-22]. This presents a significant challenge to efforts to evaluate treatment efficacy and safety in elderly patients [22]. Furthermore, there is limited knowledge about the use of recommended newer agents for the treatment of mCRC in community settings, particularly for older and demographically diverse patient populations [23]. However, there is evidence to suggest variations in the management of patients with all stages of CRC with several studies reporting lower rates of chemotherapy for older patients [23-27] and almost $30 \%$ of stage III and IV patients were less likely to receive guideline-recommended therapies [27]. An analysis of patients in the National Cancer Data Base who were treated for CRC from 2003 to 2007 revealed that $25.9 \%$ of patients with stage IV disease received no chemotherapy and older patients with preexisting comorbid conditions were at increased risk of under-treatment [23]. Comorbid health conditions and older age appear to influence physicians' choice of treatment regimen for all stages of CRC with older patients more likely to receive shorter chemotherapy regimens with less toxicity [23-27]. The goal of this study was to evaluate treatment patterns, OS, and frequency of complications requiring medical resource utilization in older, demographically diverse patients undergoing treatment for mCRC.

\section{Methods}

\section{Data Sources}

We utilized population-based claims data from the Surveillance, Epidemiology, and End Results (SEER)-Medicare linked database. The SEER-Medicare database is a collaborative effort of the National Cancer Institute, the SEER registries, and the Centers for Medicare \& Medicaid Services. As detailed elsewhere [28], this database provides information on Medicare patients included in SEER, a collection of 18 population-based cancer registries of incident cases from diverse geographic areas representative of approximately $28 \%$ of the US population. All incident cancer patients reported to SEER registries are crossmatched with a master file of Medicare enrollment [29]. Approximately $97 \%$ of persons 65 years or older are eligible for Medicare with all beneficiaries eligible for part A coverage including inpatient care, skilled nursing, home healthcare, and hospice care. Approximately $95 \%$ of beneficiaries also subscribe to part $\mathrm{B}$, which covers physician services and outpatient care. The SEER-Medicare linkage includes all Medicare-eligible persons in the SEER database through 2007 and their Medicare claims for part A (inpatient care) and part B (outpatient and physician services) through 2009. Institutional review board approval for this study was waived because the SEER-Medicare database does not include personal identifiers.

\section{Study Population}

Eligibility criteria for study inclusion included: (1) a first primary diagnosis of stage IIIB, IIIC, or IV CRC from January 1, 2000 through December 31, 2007, (2) age $\geq 66$ years, (3) treatment with any oral or infused chemotherapy after diagnosis, and (4) survival time $\geq 60$ days following the date of first-line chemotherapy initiation. We eliminated patients whose survival was less than 60 days to minimize the introduction of immortal time bias into the analyses [30]. Patients were also excluded if their date of death was recorded prior to or in the same month as diagnosis, enrollment in Medicare parts A and B for less than 12 months before the diagnosis date, enrollment in a health maintenance organization (HMO) for any period of the 12 months prior to diagnosis (because data were unavailable for this time), two or more claims for chemotherapy prior to diagnosis (to ensure that the cases were previously untreated), and finally, cases were excluded if they underwent primary resection of the tumor prior to initiating chemotherapy (to eliminate potential adjuvant cases). See Supplementary Fig. 1 for schematic of inclusion/exclusion process.

\section{Study Variables}

The SEER program routinely collects data on patient demographics including age, race/ethnicity, residence, and socioeconomic status (income and education per census tract), primary tumor site, tumor morphology, stage at diagnosis, first course of treatment, and follow-up for vital status. Median annual household income at the census tract level and percentage of the adult population who completed specific levels of education at the zip code level were used as a proxy for socioeconomic status. SEER site codes identified colon and rectum cancer cases. The American Joint 
Fig 1 Type of treatment by year of initiation

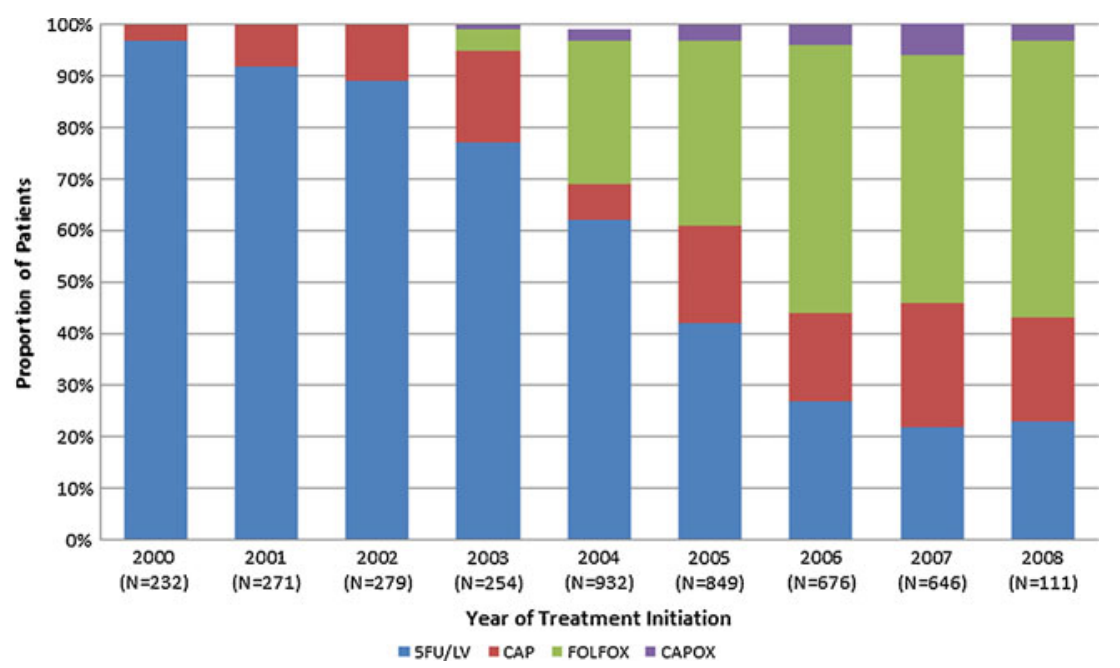

Committee on Cancer and SEER stage groupings were used to identify stage at diagnosis.

To identify claims for chemotherapy administration, [31] data were abstracted from four merged SEER-Medicare claims files including (1) Medicare provider analysis and review, (2) carrier claims from the National Claims History, (3) outpatient claims (OUTSAF), and (4) durable medical equipment (DME). Claims for oral equivalents of intravenous chemotherapies (i.e., capecitabine) were identified in the DME file. Chemotherapy agents were characterized and quantified using International Classification of Disease (ICD) diagnosis codes, ICD procedural codes, Current Procedural Terminology codes, Healthcare Common Procedural Coding System (HCPCS) codes, and revenue center codes. Chemotherapy claims were searched for specific drug codes to identify the type of chemotherapy used. The absence of these claims indicated lack of treatment. The first chemotherapy claim following diagnosis indicated the start of therapy. Patients were classified into one of four treatment groups (5-FU/LV, CAP, FOLFOX, and CAPOX) based on all chemotherapy administered during the first 60 days after treatment initiation.

Medicare claims identified patients who underwent primary resection of the tumor prior to initiating chemotherapy. Surgical procedures included hemicolectomy, subtotal colectomy, and total colectomy. Claims filed 1 year before diagnosis were used to determine baseline comorbidity burden. Comorbidities were aggregated to formulate the National Cancer Institute (NCI) comorbidity index, a revised version of the Charlson comorbidity index [32]. The incidence of specific treatment-related complications (anemia, neutropenia, nausea/vomiting, diarrhea, and dehydration) requiring medical resource utilization was assessed 180 days following treatment initiation. This 180 -day period was selected as appropriate based on the National Comprehensive Cancer Network guidelines that recommend 6 months of adjuvant treatment for stage II and III CRC or for stage
IV stable disease [33]. Anemia was defined by the condition-specific ICD-9 diagnosis codes, a revenue center code or HCPCS code for a red blood cell transfusion, or a revenue center code or J-code for an erythropoiesisstimulating agent. Other treatment complications were defined using the condition-specific ICD-9 codes in both inpatient and outpatient Medicare claims records (codes available upon request).

The date of death was determined by using the Medicare date or the SEER date of death if the Medicare date was missing. All other patients were assumed to be alive at the end of the follow-up period on December 31, 2009, although they may have been censored earlier for other reasons such as development of a second primary cancer or Medicare claims no longer available.

\section{Statistical Analysis}

All statistical analyses were performed using SAS software, version 9.1.3 (SAS Institute Inc., Cary, NC). Statistical comparisons were made between 5 -FU/LV vs. CAP and FOLFOX vs. CAPOX. Descriptive statistics were calculated for demographic and clinical variables and treatment patterns. Differences between treatment groups were evaluated with chi-square tests for categorical variables and analysis of variance or $t$ test for continuous variables. A $P$ value $<0.05$ was considered statistically significant.

In the survival analysis to assess overall risk of death, we compared two approaches as a sensitivity exercise: (1) multivariate Cox proportional hazards regression and (2) propensity score-weighted Cox proportional hazards regression. In the first model, we adjusted for confounders that were selected from demographic and clinical characteristics using the backward elimination strategy [34]. In the second model, multinomial logistic regression was used to calculate a propensity score for each individual. The propensity score is the conditional probability of each patient 
receiving a specific treatment based on baseline characteristics [35]. The effect of the propensity score weights was to balance the groups to reduce potential bias associated with treatment selection. A propensity score-weighted Cox proportional hazards regression model was fitted to compare overall survival between treatment groups. Follow-up was calculated beginning on the date of treatment initiation up until the first occurrence of a censoring event: date of death, development of a second primary tumor, last date for which Medicare claims were available, or last date of the follow-up period (December 31, 2009).

\section{Results}

Demographic and Clinical Characteristics

Of the 7,061 patients who met all study inclusion criteria, 2,213 were treated with 5-FU/LV, 1,298 received FOLFOX, 617 were administered with CAP, and 122 received CAPOX (Table 1). Of the remaining 2,811 patients, about $28 \%$ received irinotecan-based therapy, $57 \%$ received other types of chemotherapy, and $15 \%$ received an unknown type of chemotherapy. Compared with patients administered with 5-FU/LV, patients treated with CAP were older, more likely female, diagnosed with stage IIIB/C disease, and had higher tumor grade. A higher proportion of patients treated with CAPOX were older and had stage IV disease compared with those treated with FOLFOX. Higher rates of treatment with CAP and CAPOX were evident for patients residing in the west and those with higher levels of income and education compared with patients administered with 5-FU/LV and FOLFOX, respectively.

\section{Treatment Patterns}

Use of CAP, CAPOX, and FOLFOX increased over time while treatment with 5-FU/LV decreased during the same time period (Fig. 1). The mean time to initiation of chemotherapy following diagnosis was similar between treatment groups ranging from 74 days for 5-FU/LV, 77 days for CAPOX, 78 days for FOLFOX, and 81 days for CAP (Table 2). The mean duration of treatment was longer for those administered with 5-FU/LV (147 days) compared with 128 days for the CAP group $(P<0.0001)$ while there was no significant difference in duration of treatment with CAPOX (143 days) and FOLFOX (151 days; $P=0.2335$ ).

\section{Complications Requiring Medical Resource Utilization}

The overall rate of complications requiring medical resource utilization (Table 3) within 180 days after treatment initiation was higher for patients treated with 5-FU/LV (54.3\%) and FOLFOX (74.9\%) compared with CAP (17.2\%) and CAPOX $(56.6 \%)$, respectively $(<0.0001$ for both comparisons). The three most frequent complications requiring medical resource utilization were anemia, nausea/vomiting, and diarrhea with significantly higher rates for $5-\mathrm{FU} / \mathrm{LV}$ vs. CAP and FOLFOX vs. CAPOX $(P<0.0001$ for all comparisons).

\section{Survival Outcomes}

The median survival time was 32.6 months ( $95 \%$ CI, $28.1-$ 38.8 ) in the CAP group and 31.9 months ( $95 \%$ CI, 29.1-34.9) in the 5-FU/LV group (log rank $P=0.6683$; Fig. 2$)$. The multivariate Cox regression survival analysis (Table 4) revealed no significant differences in risk of death between CAP compared with 5-FU/LV (HR, 0.919; $95 \%$ CI, 0.799 $1.058 ; P=0.2396)$. This finding was confirmed in the propensity-weighted Cox regression. The full Cox model included treatment, age, sex, race, positive lymph nodes, tumor grade, comorbidity score, geographic region, and income. After backward elimination, age, greater number of positive lymph nodes, higher tumor grade, and lower income levels were identified as significant predictors of mortality.

Figure 3 demonstrates that while the median survival time was not reached, the 3-year unadjusted survival rates for CAPOX and FOLFOX were $71.6 \%$ (95 \% CI, 54.183.3 ) and $68.5 \%$ (95\% CI, 64.2-72.3), respectively (log rank $P=0.6737$ ). There were no significant differences in adjusted overall survival between CAPOX and FOLFOX (HR, 1.047; $95 \%$ CI, 0.676-1.622; $P=0.8367$ ). The propensity-weighted Cox regression analysis also confirmed these findings (Table 5). The full Cox model included treatment, age, sex, race, positive lymph nodes, tumor grade, comorbidity score, geographic region, and income. After backward elimination, age and tumor grade maintained statistical significance in the model.

\section{Discussion}

Clinical trials confirm that therapy with agents including 5FU, oxaliplatin, capecitabine, and oxaliplatin is associated with improved survival of patients with stage III and IV mCRC [3-5, 7, 8, 10-16, 18, 36-43]. Importantly, compared with younger patients in the setting of clinical trials, this population-based retrospective cohort analysis of elderly patients in community settings revealed comparable benefits in overall survival and complications requiring medical resource utilization in response to these treatments [44, 45].

The finding that patients treated with CAP were older and had a higher comorbidity burden compared with the three other treatment groups may reflect a belief among physicians that elderly patients are frailer and less able to tolerate 
Table 1 Demographic and clinical characteristics at baseline

\begin{tabular}{|c|c|c|c|c|c|c|c|c|c|c|}
\hline \multirow[t]{2}{*}{ Characteristic } & \multicolumn{2}{|c|}{$\mathrm{CAP}(N=617)$} & \multicolumn{2}{|c|}{ 5-FU/LV $(N=2,213)$} & \multirow[t]{2}{*}{$P$ value } & \multicolumn{2}{|c|}{ CAPOX $(N=122)$} & \multicolumn{2}{|c|}{ FOLFOX $(N=1,298)$} & \multirow[t]{2}{*}{$P$ value } \\
\hline & $n$ & $\%$ & $n$ & $\%$ & & $n$ & $\%$ & $n$ & $\%$ & \\
\hline \multicolumn{11}{|l|}{ Age at diagnosis } \\
\hline $66-70$ & 96 & 15.6 & 503 & 22.7 & \multirow[t]{4}{*}{$<0.0001$} & 43 & 35.2 & 495 & 38.1 & \multirow[t]{4}{*}{0.0924} \\
\hline $71-75$ & 137 & 22.2 & 641 & 29.0 & & 30 & 24.6 & 419 & 32.3 & \\
\hline $76-80$ & 160 & 25.9 & 591 & 26.7 & & 38 & 31.1 & 296 & 22.8 & \\
\hline$>80$ & 224 & 36.3 & 478 & 21.6 & & 11 & 9.0 & 88 & 6.8 & \\
\hline \multicolumn{11}{|l|}{ Sex } \\
\hline Male & 243 & 39.4 & 1,029 & 46.5 & \multirow[t]{2}{*}{0.0017} & 56 & 45.9 & 632 & 48.7 & \multirow[t]{2}{*}{0.5557} \\
\hline Female & 374 & 60.6 & 1,184 & 53.5 & & 66 & 54.1 & 666 & 51.3 & \\
\hline \multicolumn{11}{|l|}{ Race/ethnicity } \\
\hline White & 499 & 80.9 & 1,850 & 83.6 & \multirow[t]{2}{*}{0.1115} & 104 & 85.2 & 1,130 & 87.1 & \multirow[t]{2}{*}{0.5708} \\
\hline Non-White & 118 & 19.1 & 363 & 16.4 & & 18 & 14.8 & 168 & 12.9 & \\
\hline \multicolumn{11}{|l|}{ Stage at diagnosis } \\
\hline Stage IIIB/C & 381 & 61.8 & 1,027 & 46.4 & \multirow[t]{2}{*}{$<0.0001$} & 79 & 64.8 & 962 & 74.1 & \multirow[t]{2}{*}{0.0254} \\
\hline Stage IV & 236 & 38.2 & 1,186 & 53.6 & & 43 & 35.2 & 336 & 25.9 & \\
\hline \multicolumn{11}{|l|}{ Tumor grade } \\
\hline Grade 1 & 33 & 5.3 & 96 & 4.3 & \multirow[t]{5}{*}{0.0145} & $81^{\mathrm{a}}$ & 66.4 & 69 & 5.3 & \multirow[t]{5}{*}{0.9117} \\
\hline Grade 2 & 351 & 56.9 & 1,397 & 63.1 & & & & 790 & 60.9 & \\
\hline Grade 3 & 189 & 30.6 & 617 & 27.9 & & $41^{\mathrm{a}}$ & 33.6 & 373 & 28.7 & \\
\hline Grade 4 & 18 & 2.9 & 33 & 1.5 & & & & 27 & 2.1 & \\
\hline Unknown & 26 & 4.2 & 70 & 3.2 & & & & 39 & 3.0 & \\
\hline \multicolumn{11}{|l|}{ Comorbidity score } \\
\hline 0 & 338 & 54.8 & 1,307 & 59.1 & 0.1999 & 78 & 63.9 & 806 & 62.1 & 0.9469 \\
\hline 1 & 173 & 28.0 & 562 & 25.4 & & 32 & 26.2 & 342 & 26.3 & \\
\hline 2 & 60 & 9.7 & 213 & 9.6 & & $12^{\mathrm{a}}$ & 9.9 & 97 & 7.5 & \\
\hline$\geq 3$ & 46 & 7.5 & 131 & 5.9 & & & & 53 & 4.1 & \\
\hline Geographic region & & & & & & & & & & \\
\hline Midwest & 81 & 13.1 & 261 & 11.8 & 0.0001 & $17^{\mathrm{a}}$ & 13.9 & 170 & 13.1 & $<0.0001$ \\
\hline Northeast & 34 & 5.5 & 145 & 6.6 & & & & 85 & 6.5 & \\
\hline South & 251 & 40.7 & 1,102 & 49.8 & & 30 & 24.6 & 567 & 43.7 & \\
\hline West & 251 & 40.7 & 705 & 31.9 & & 75 & 61.5 & 476 & 36.7 & \\
\hline Median income quartiles & & & & & & & & & & \\
\hline 1 -low & 130 & 21.1 & 576 & 26.0 & 0.0002 & 21 & 17.2 & 334 & 25.7 & 0.1633 \\
\hline 2 & 145 & 23.5 & 559 & 25.3 & & 35 & 28.7 & 319 & 24.6 & \\
\hline 3 & 146 & 23.7 & 557 & 25.2 & & 30 & 24.6 & 325 & 25.0 & \\
\hline 4-high & 195 & 31.6 & 509 & 23.0 & & 36 & 29.5 & 318 & 24.5 & \\
\hline Education & Mean & $95 \% \mathrm{CI}$ & Mean & $95 \% \mathrm{CI}$ & $P$ value & Mean & $95 \% \mathrm{CI}$ & Mean & $95 \% \mathrm{CI}$ & $P$ value \\
\hline Less than high school, $\%$ & 18.7 & {$[17.73,19.76]$} & 20.0 & {$[19.46,20.46]$} & 0.0298 & 17.1 & {$[14.84,19.41]$} & 18.5 & {$[17.92,19.17]$} & 0.1966 \\
\hline High school only, \% & 26.7 & {$[25.89,27.43]$} & 28.7 & {$[28.33,29.10]$} & $<0.0001$ & 23.1 & {$[21.36,24.88]$} & 28.1 & {$[27.54,28.57]$} & $<0.0001$ \\
\hline Some college, $\%$ & 27.5 & {$[26.91,28.00]$} & 27.1 & {$[26.87,27.43]$} & 0.3359 & 28.6 & {$[27.25,29.91]$} & 28.0 & {$[27.66,28.41]$} & 0.4058 \\
\hline At least a college degree, $\%$ & 27.1 & {$[25.81,28.47]$} & 24.2 & {$[23.55,24.81]$} & $<0.0001$ & 31.2 & {$[27.99,34.36]$} & 25.4 & {$[24.50,26.24]$} & 0.0002 \\
\hline
\end{tabular}

${ }^{\text {a }}$ Cells with counts of less than 11 are combined in compliance with the National Cancer Institute data use agreement for small cell sizes

aggressive or more toxic treatments. A recent review of the medical records of patients aged 65 or older diagnosed with stage III colon cancer between 2003 and 2006 revealed that $61 \%$ received a regimen containing oxaliplatin, $54 \%$ were treated with FOLFOX, $19 \%$ received 5-FU/LV, and $12 \%$ were administered with capecitabine monotherapy. Among those not treated with oxaliplatin, the primary reason was comorbid health conditions with age cited as a reason for not administering oxaliplatin for $19 \%$ of patients [27]. 
Table 2 Time to first-line treatment and duration of first-line treatment

\begin{tabular}{|c|c|c|c|c|c|c|c|}
\hline & $N$ & Mean & SD & Median & Min & Max & $P$ value \\
\hline \multicolumn{8}{|c|}{ Time to treatment ${ }^{\mathrm{a}}$, days } \\
\hline CAP & 545 & 80.68 & 34.93 & 74 & 7 & 179 & \multirow[t]{2}{*}{$<0.0001$} \\
\hline 5-FU/LV & 2,117 & 73.80 & 29.63 & 69 & 1 & 177 & \\
\hline CAPOX & 120 & 77.11 & 33.68 & 72 & 15 & 175 & \multirow[t]{2}{*}{0.8811} \\
\hline FOLFOX & 1,255 & 77.53 & 29.18 & 72 & 14 & 175 & \\
\hline \multicolumn{8}{|c|}{ Duration of treatment $\mathrm{t}^{\mathrm{b}}$, days } \\
\hline CAP & 603 & 128.41 & 76.91 & 118 & 30 & 357 & \multirow[t]{2}{*}{$<0.0001$} \\
\hline 5-FU/LV & 2,140 & 147.48 & 75.01 & 143 & 30 & 365 & \\
\hline CAPOX & 119 & 143.43 & 64.45 & 141 & 31 & 300 & \multirow[t]{2}{*}{0.2335} \\
\hline FOLFOX & 1,285 & 150.93 & 65.78 & 157 & 30 & 360 & \\
\hline
\end{tabular}

a Time to treatment initiation defined as "time from diagnosis" to "date of first chemotherapy claim"

${ }^{\mathrm{b}}$ Duration of treatment defined as time from date of first chemotherapy claim to 30 days following last administration of first-line agent, or to the day prior to second-line treatment initiation or 30 days following last administration of first-line agent if gap in therapy is $>90$ days

Patient characteristics such as age, gender, race, and comorbidity burden appear to be important factors in prescribing chemotherapy treatment, but after adjusting for these factors, there were no significant differences in OS between the CAP-based and 5-FU/LV-based regimens. This is an encouraging finding for all patients diagnosed with advanced stage CRC, suggesting that currently available and recommended systemic therapies are equally effective for patients with diverse clinical and demographic characteristics.

Our study observed that complications requiring medical resource utilization were less frequent for $\mathrm{CAP} \pm$ oxaliplatin regimens while achieving an equivalent survival benefit compared with $5-\mathrm{FU} / \mathrm{LV} \pm$ oxaliplatin regimens. This confirms similar observations from randomized clinical trials that CAP monotherapy is associated with a lower rate of adverse events and reduced medical resource utilization $[8$, 46]. Randomized clinical trials report comparative safety profiles for $\mathrm{CAP} \pm$ oxaliplatin regimens vs. $5-\mathrm{FU} / \mathrm{LV} \pm$ oxaliplatin with more grade 3 or 4 neutropenia and neutropenic fever associated with FOLFOX and more grade 3 hand-foot syndrome and grade 3 or 4 diarrhea associated with CAPOX [11, 18, 47]. However, the incidence of medically significant diarrhea, i.e., requiring medical resources, was reduced in patients receiving CAPOX vs. FOLFOX in our study. Dose selection was at the discretion of the physician and dosing information could not be determined retrospectively from available data within the claims dataset. Regional differences in tolerance to FP, both CAP and 5-FU/ $\mathrm{LV}$, have been reported with US patients more likely to experience grade 3 or 4 FP-related toxicities compared with patients from other parts of world, particularly Asia [48-50]. Physicians may have elected to use doses (lower) and/or treatment schedules other than those tested in clinical trials that may have impacted the safety profile of the regimen. These findings confirm that capecitabine-based treatments can be delivered to elderly patients under the conditions of routine medical care with outcomes similar to those achieved in overall clinical trial populations for patients $\geq 65$ years of age.

Table 3 Incidence of medical resource utilization related treatment complications requiring intervention (hospitalization or treatment) within 180 days after initiation of treatment

\begin{tabular}{|c|c|c|c|c|c|c|c|c|c|c|}
\hline \multirow[t]{2}{*}{ Adverse events } & \multicolumn{2}{|c|}{$\mathrm{CAP}(N=617)$} & \multicolumn{2}{|c|}{$5-\mathrm{FU} / \mathrm{LV}(N=2,213)$} & \multirow[t]{2}{*}{$P$ value } & \multicolumn{2}{|c|}{ CAPOX $(N=122)$} & \multicolumn{2}{|c|}{ FOLFOX $(N=1,298)$} & \multirow[t]{2}{*}{$P$ value } \\
\hline & $n$ & $\%$ & $n$ & $\%$ & & $n$ & $\%$ & $n$ & $\%$ & \\
\hline Any treatment-related complications & 106 & 17.2 & 1,201 & 54.3 & $<0.0001$ & 69 & 56.6 & 972 & 74.9 & $<0.0001$ \\
\hline Anemia & 80 & 13.0 & 879 & 39.7 & $<0.0001$ & 38 & 31.1 & 699 & 53.9 & $<0.0001$ \\
\hline Nausea/vomiting & 24 & 3.9 & 453 & 20.5 & $<0.0001$ & 36 & 29.5 & 494 & 38.1 & $<0.0001$ \\
\hline Diarrhea & 18 & 2.9 & 157 & 7.1 & $<0.0001$ & $-^{\mathrm{a}}$ & $-^{\mathrm{a}}$ & 89 & 6.9 & $<0.0001$ \\
\hline Neutropenia & $-{ }^{\mathrm{a}}$ & $-^{\mathrm{a}}$ & 13 & 0.6 & 0.0013 & $-{ }^{\mathrm{a}}$ & $-{ }^{\mathrm{a}}$ & 121 & 9.3 & $<0.0001$ \\
\hline Dehydration & $-{ }^{\mathrm{a}}$ & $-{ }^{\mathrm{a}}$ & 52 & 2.3 & $<0.0001$ & $-{ }^{\mathrm{a}}$ & $--^{\mathrm{a}}$ & 108 & 8.3 & $<0.0001$ \\
\hline
\end{tabular}

${ }^{a}$ Cells with counts of less than 11 are suppressed in compliance with the National Cancer Institute's data use agreement for small cell sizes 
Fig 2 Kaplan-Meier curve of overall survival by treatment (CAP vs. 5-FU/LV)

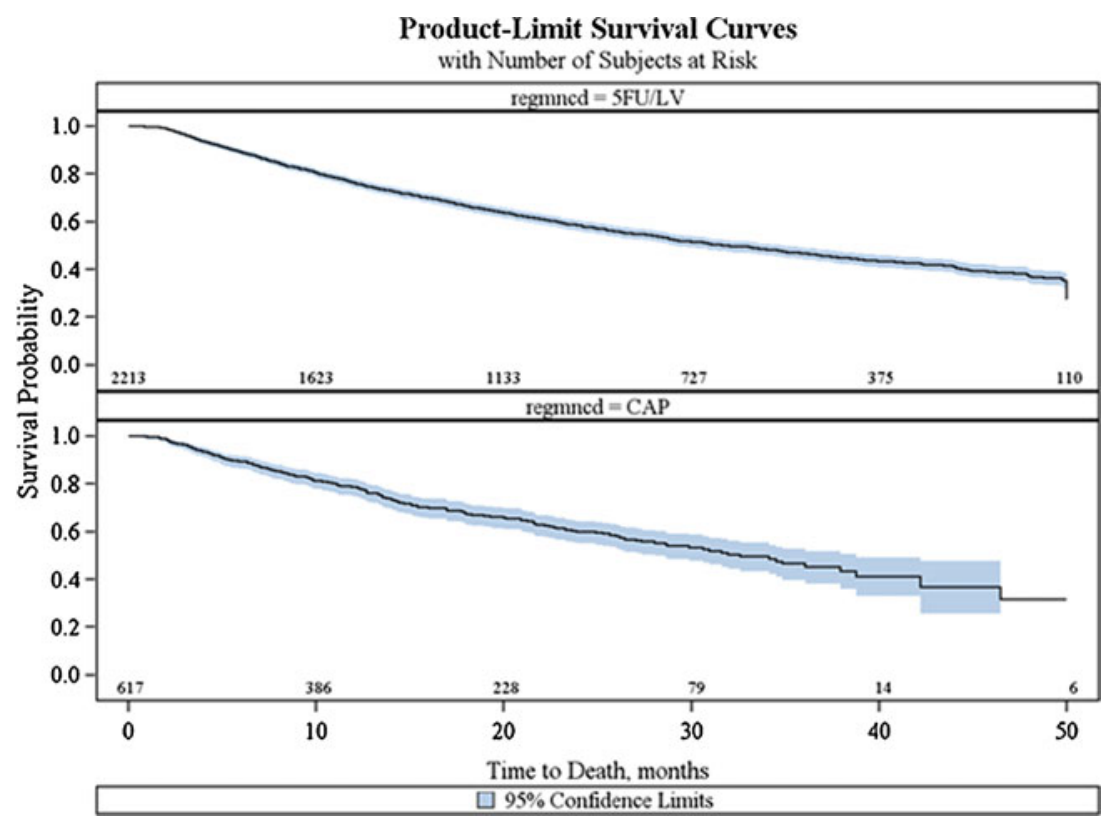

Initiation of chemotherapy for all four treatment regimens was longer (mean time, 74 to 81 days) than the typical 30 days that would be expected. Prior research has shown that not only do treatment rates decline dramatically with increasing age [51], but older age is associated with delayed chemotherapy initiation [52] and lower rates of chemotherapy completion [53]. These age disparities in treatment patterns are associated with higher mortality $[52,53]$ and

Table 4 Multivariate Cox regression of overall survival (CAP vs. 5-FU/LV)

\begin{tabular}{|c|c|c|c|c|c|c|c|}
\hline \multirow[t]{2}{*}{ Covariates } & \multirow[t]{2}{*}{$N$} & \multicolumn{3}{|c|}{ Multivariate Cox regression reduced model ${ }^{\mathrm{a}}$} & \multicolumn{3}{|c|}{ Propensity weighted Cox regression ${ }^{\mathrm{b}}$} \\
\hline & & HR & $95 \% \mathrm{CI}$ & $P$ value & HR & $95 \% \mathrm{CI}$ & $P$ value \\
\hline \multicolumn{8}{|l|}{ Treatment } \\
\hline 5-FU/LV (ref) & 2,213 & 1.000 & & & 1.000 & & \\
\hline CAP & 617 & 0.919 & $0.799-1.058$ & 0.2396 & 0.868 & $0.753-0.999$ & 0.0487 \\
\hline \multicolumn{8}{|l|}{ Age at diagnosis } \\
\hline $66-70$ (ref) & 599 & 1.000 & & & & & \\
\hline $71-75$ & 778 & 1.078 & $0.930-1.250$ & 0.3184 & & & \\
\hline $76-80$ & 751 & 1.310 & $1.131-1.519$ & 0.0003 & & & \\
\hline$>80$ & 702 & 1.455 & $1.250-1.694$ & $<0.0001$ & & & \\
\hline \multicolumn{8}{|c|}{ Positive lymph nodes } \\
\hline 0 (ref) & 278 & 1.000 & & & & & \\
\hline $1-3$ & 1,362 & 0.710 & $0.599-0.841$ & $<0.0001$ & & & \\
\hline$\geq 4$ & 1,024 & 1.275 & $1.077-1.508$ & 0.0048 & & & \\
\hline \multicolumn{8}{|l|}{ Tumor grade } \\
\hline $1-3$ (ref) & 1,877 & 1.000 & & & & & \\
\hline $3-4$ & 857 & 1.253 & $1.123-1.398$ & $<0.0001$ & & & \\
\hline \multicolumn{8}{|c|}{ Median income quartiles } \\
\hline 1 (low) (ref) & 768 & 1.000 & & & & & \\
\hline 2 & 720 & 0.949 & $0.825-1.092$ & 0.4668 & & & \\
\hline 3 & 656 & 0.858 & $0.742-0.993$ & 0.0402 & & & \\
\hline 4 (high) & 673 & 0.909 & $0.787-1.049$ & 0.1927 & & & \\
\hline
\end{tabular}

${ }^{a}$ Reduced model by backward elimination. Full model included age, sex, race, positive lymph nodes, tumor grade, comorbidity score, geographic region, and income

${ }^{\mathrm{b}}$ Propensity score weighted for age, sex, race, positive lymph nodes, tumor grade, comorbidity score, geographic region, and income 
Fig 3 Kaplan-Meier curve of overall survival by treatment (CAPOX vs. FOLFOX)

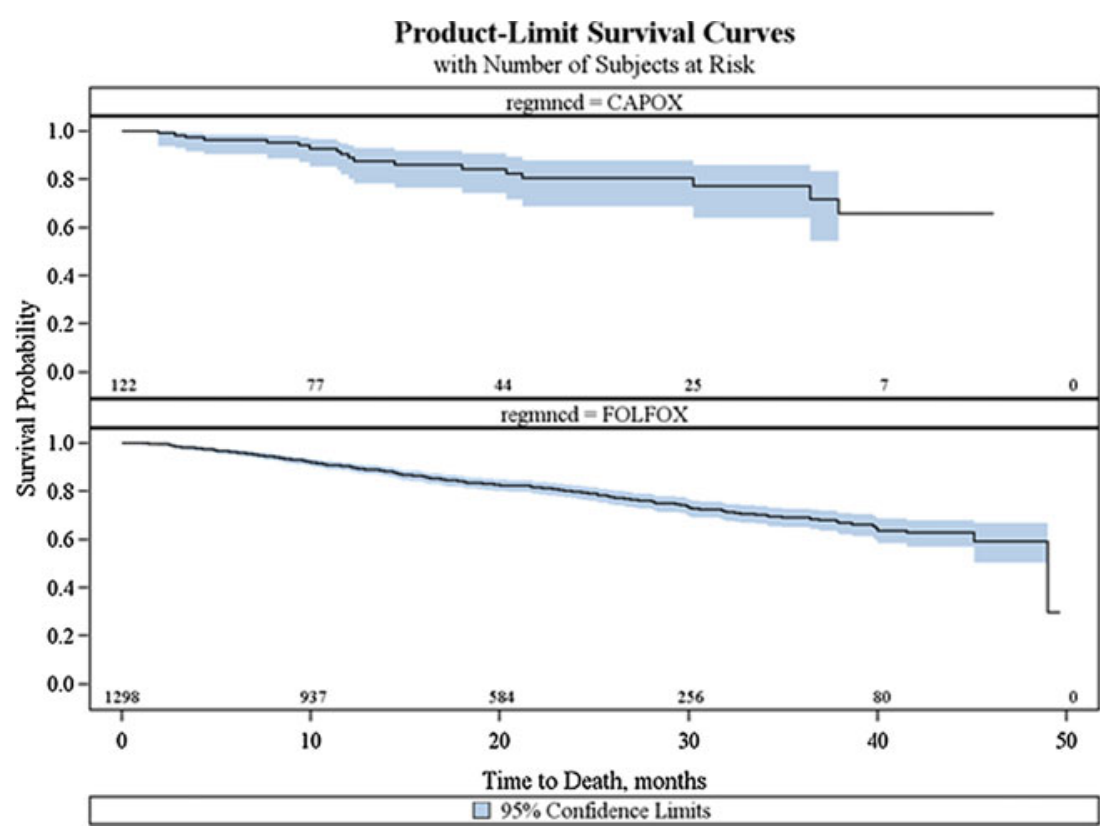

our results provide further support that demographic factors such as age should not discourage the use of guidelinerecommended therapies.

\section{Study Strengths and Limitations}

This study has several strengths, including the large sample size from a population-based registry with a wide geographic representation of patients with CRC in the USA. The SEER-Medicare dataset provides inpatient and outpatient data, comprehensive information about covered services, all claims regardless of residence or care out of area, and longitudinal data with claims for services from the time a person is eligible for Medicare until the date of death. However, use of the SEERMedicare data for this type of analysis has some limitations, particularly for determining accurate utilization rates of oral chemotherapeutic agents such as capecitabine. A recent comparison of Medicare claims with the National Cancer Institute's Patterns of Care studies showed that among patients with various cancers receiving chemotherapy (including stage II/III CRC), Medicare claims data more accurately identified agents that were intravenously administered [54].

Table 5 Multivariate Cox regression of overall survival (CAPOX vs. FOLFOX)

\begin{tabular}{|c|c|c|c|c|c|c|c|}
\hline \multirow[t]{2}{*}{ Covariates } & \multirow[t]{2}{*}{$N$} & \multicolumn{3}{|c|}{ Multivariate Cox regression reduced model ${ }^{\mathrm{a}}$} & \multicolumn{3}{|c|}{ Propensity weighted Cox regression ${ }^{\mathrm{b}}$} \\
\hline & & HR & $95 \% \mathrm{CI}$ & $P$ value & HR & $95 \% \mathrm{CI}$ & $P$ value \\
\hline \multicolumn{8}{|l|}{ Treatment } \\
\hline FOLFOX (ref) & 1,298 & 1.000 & & & 1.000 & & \\
\hline CAPOX & 122 & 1.047 & $0.676-1.622$ & 0.8367 & 1.129 & $0.749-1.699$ & 0.5626 \\
\hline \multicolumn{8}{|l|}{ Age at diagnosis } \\
\hline $66-70$ (ref) & 538 & 1.000 & & & & & \\
\hline $71-75$ & 449 & 1.056 & $0.795-1.402$ & 0.7071 & & & \\
\hline $76-80$ & 334 & 1.207 & $0.892-1.633$ & 0.2217 & & & \\
\hline$>80$ & 99 & 1.731 & $1.138-2.633$ & 0.0104 & & & \\
\hline \multicolumn{8}{|l|}{ Tumor grade } \\
\hline $1-3$ (ref) & 940 & 1.000 & & & & & \\
\hline $3-4$ & 437 & 1.678 & $1.318-2.137$ & $<0.0001$ & & & \\
\hline
\end{tabular}

${ }^{a}$ Reduced model by backward elimination. Full model included age, sex, race, positive lymph nodes, tumor grade, comorbidity score, geographic region, and income

${ }^{\mathrm{b}}$ Propensity score weighted for age, sex, race, positive lymph nodes, tumor grade, comorbidity score, geographic region, and income 
In addition, the SEER-Medicare database does not provide data on performance status or lifestyle factors, such as smoking. These factors could have affected the treatment patterns we observed or clinicians' initial decisions to treat these patients. Furthermore, treatment patterns for the older population with Medicare coverage may be different from those used for younger patients and, therefore, the results might have limited applicability to younger populations in real-world settings. This analysis also does not yield information about patients enrolled in HMOs since these data are not collected by Medicare. It is conceivable that treatment patterns, prognosis, and complications may differ between HMO and Medicare enrollees. Previous studies found that Medicare HMO enrollees with colon cancer had better OS compared with fee-forservice (FFS) plan members $[55,56]$. These mortality differences might have been due to higher use of screening and preventive services for HMO patients or the possibility that HMO enrollees tend to be healthier than FFS enrollees.

\section{Conclusions}

Overall survival for elderly mCRC patients who were treated under conditions of routine medical oncology practice was comparable between CAP and 5-FU/LV and between CAPOX and FOLFOX. These results are consistent with those reported among younger patients in randomized clinical trials. The rate of treatment-related complications requiring medical resource use was lower for patients administered capecitabine monotherapy and in combination with oxaliplatin compared with 5FU/LV and FOLFOX, respectively. These findings confirm that capecitabine-based regimens are an appropriate treatment choice for elderly patients with mCRC. These data also offer support for the use of treatments for elderly patients that are consistent to those administered to younger patients and imply that age should not discourage the use of guidelinerecommended therapies for $\mathrm{mCRC}$.

Further research is required to evaluate patterns and outcomes of care for patients with varying performance status since this information is not included in the SEER-Medicare database and we were unable to examine possible interactions between performance status and prognosis and the incidence of treatment-related complications. Another area that warrants further research is a comparison of the treatment patterns, prognosis, and complications of patients enrolled in HMOs compared with those in FFS plans.

Acknowledgments Funding for this study was provided by Genentech, Inc. This study used the linked SEER-Medicare database. We acknowledge the efforts of the Applied Research Program, NCI (Bethesda, MD), the Office of Information Services and the Office of Strategic Planning, Health Care Financing Administration (Baltimore, MD), Information Management Services, Inc. (Silver Spring, MD), and the SEER Program tumor registries in the creation of the SEER-
Medicare database. The interpretation and reporting of these data are the sole responsibility of the authors. The authors acknowledge the assistance of Carole Alison Chrvala, PhD, of Health Matters, Inc. for her assistance with the writing and editing of this manuscript.

Conflict of interest Dr. Satram-Hoang is an employee of Q.D. Research, Inc. and serves as a consultant to Genentech, Inc. Drs. Lee, McKenna, and Reyes are employees of Genentech Inc. Dr. Yu is a consultant to Genentech, Inc. The remaining authors declare no competing financial interests.

Open Access This article is distributed under the terms of the Creative Commons Attribution License which permits any use, distribution, and reproduction in any medium, provided the original author(s) and the source are credited.

\section{References}

1. Howlader N, et al. SEER Cancer Statistics Review, 1975-2008. 2011 [based on November 2010 SEER data submission, posted to the SEER web site 2011]. Available from: http://seer.cancer.gov/ csr/1975_2008/. Accessed 6 Feb 2012.

2. Siegel R, Naishadham D, Jemal A. Cancer statistics, 2012. CA Cancer J Clin. 2012;1:10-29.

3. Douillard JY, Bennouna J, Senellart H. Is XELOX equivalent to FOLFOX or other continuous-infusion 5-fluorouracil chemotherapy in metastatic colorectal cancer? Clin Color Cancer. 2008;3:206-11.

4. Koukourakis GV, et al. Efficacy of the oral fluorouracil pro-drug capecitabine in cancer treatment: a review. Molecules. 2008;8:1897-922.

5. Thirion P, et al. Modulation of fluorouracil by leucovorin in patients with advanced colorectal cancer: an updated metaanalysis. J Clin Oncol. 2004;18:3766-75.

6. Goldberg RM, et al. Randomized controlled trial of reduced-dose bolus fluorouracil plus leucovorin and irinotecan or infused fluorouracil plus leucovorin and oxaliplatin in patients with previously untreated metastatic colorectal cancer: a North American Intergroup Trial. J Clin Oncol. 2006;21:3347-53.

7. Braun $\mathrm{AH}$, et al. New systemic frontline treatment for metastatic colorectal carcinoma. Cancer. 2004;8:1558-77.

8. Van Cutsem E, et al. Oral capecitabine compared with intravenous fluorouracil plus leucovorin in patients with metastatic colorectal cancer: results of a large phase III study. J Clin Oncol. 2001;21:4097-106.

9. Van Cutsem E, et al. Oral capecitabine vs intravenous 5-fluorouracil and leucovorin: integrated efficacy data and novel analyses from two large, randomised, phase III trials. Br J Cancer. 2004;6:1190-7.

10. Welles L, Hochster H, Ramanathan R, et al. Preliminary results of a randomized study of the safety and tolerability of three oxaliplatin-based regimens as first-line treatment for advanced colorectal cancer (CRC) ("TREE" study). Proc Am Soc Clin Oncol. 2004;23:254 (Abstract \#3537).

11. Cassidy J, Clarke S, and Diaz-Rubio E. First efficacy and safety results from XELOX-I/NO16966, a randomized $2 \times 2$ factorial phase III trial of XELOX vs. FOLFOX $4+$ bevacizumab or placebo in first-line metastatic colorectal cancer (MCRC), in Annual Meeting of the European Society of Medical Oncology2006: Istanbul, Turkey.

12. Ducreux M, et al. Capecitabine plus oxaliplatin (XELOX) versus 5-fluorouracil/leucovorin plus oxaliplatin (FOLFOX-6) as firstline treatment for metastatic colorectal cancer. Int $\mathrm{J}$ Cancer. 2011;128(3):682-90.

13. Diaz-Rubio E, et al. Phase III study of capecitabine plus oxaliplatin compared with continuous-infusion fluorouracil plus oxaliplatin as 
first-line therapy in metastatic colorectal cancer: final report of the Spanish Cooperative Group for the Treatment of Digestive Tumors Trial. J Clin Oncol. 2007;27:4224-30.

14. Porschen R, et al. Phase III study of capecitabine plus oxaliplatin compared with fluorouracil and leucovorin plus oxaliplatin in metastatic colorectal cancer: a final report of the AIO Colorectal Study Group. J Clin Oncol. 2007;27:4217-23.

15. Comella P, et al. Biweekly oxaliplatin combined with oral capecitabine (OXXEL regimen) as first-line treatment of metastatic colorectal cancer patients: a Southern Italy Cooperative Oncology Group phase II study. Cancer Chemother Pharmacol. 2005;5:481-6.

16. Comella P, et al. Capecitabine plus oxaliplatin for the first-line treatment of elderly patients with metastatic colorectal carcinoma: final results of the Southern Italy Cooperative Oncology Group Trial 0108. Cancer. 2005;2:282-9.

17. Goldberg RM, et al. A randomized controlled trial of fluorouracil plus leucovorin, irinotecan, and oxaliplatin combinations in patients with previously untreated metastatic colorectal cancer. J Clin Oncol. 2004;1:23-30.

18. Cassidy J, et al. XELOX vs FOLFOX-4 as first-line therapy for metastatic colorectal cancer: NO16966 updated results. Br J Cancer. 2011;1:58-64.

19. Arkenau HT, et al. Efficacy of oxaliplatin plus capecitabine or infusional fluorouracil/leucovorin in patients with metastatic colorectal cancer: a pooled analysis of randomized trials. J Clin Oncol. 2008;36:5910-7.

20. Hutchins LF, et al. Underrepresentation of patients 65 years of age or older in cancer-treatment trials. N Engl J Med. 1999;27:2061-7.

21. Murthy VH, Krumholz HM, Gross CP. Participation in cancer clinical trials: race-, sex-, and age-based disparities. JAMA. 2004;22:2720-6.

22. Townsley CA, Selby R, Siu LL. Systematic review of barriers to the recruitment of older patients with cancer onto clinical trials. J Clin Oncol. 2005;13:3112-24.

23. Chagpar R, et al. Adherence to stage-specific treatment guidelines for patients with colon cancer. J Clin Oncol. 2012;9:972-9.

24. Schrag D, et al. Age and adjuvant chemotherapy use after surgery for stage III colon cancer. J Natl Cancer Inst. 2001;11:850-7.

25. Ayanian JZ, et al. Use of adjuvant chemotherapy and radiation therapy for colorectal cancer in a population-based cohort. J Clin Oncol. 2003;7:1293-300.

26. Kahn KL, et al. Adjuvant chemotherapy use and adverse events among older patients with stage III colon cancer. JAMA. 2010;11:1037-45.

27. O'Grady MA, et al. Assessing compliance with national comprehensive cancer network guidelines for elderly patients with stage III colon cancer: the Fox Chase Cancer Center Partners' initiative. Clin Colorectal Cancer. 2011;2:113-6.

28. Warren JL, et al. Overview of the SEER-Medicare data: content, research applications, and generalizability to the United States elderly population. Med Care. 2002;8(Suppl:IV):3-18.

29. Potosky AL, et al. Potential for cancer related health services research using a linked Medicare-tumor registry database. Med Care. 1993;8:732-48.

30. Suissa S. Immortal time bias in pharmaco-epidemiology. Am J Epidemiol. 2008;4:492-9.

31. Warren JL, et al. Utility of the SEER-Medicare data to identify chemotherapy use. Med Care. 2002;8(Suppl:IV):55-61.

32. Klabunde $\mathrm{CN}$, et al. A refined comorbidity measurement algorithm for claims-based studies of breast, prostate, colorectal, and lung cancer patients. Ann Epidemiol. 2007;8:584-90.

33. NCCN, N.C.C.N, Clinical practice guidelines in oncology. $<$ http:// www.nccn.org/professionals/physician_gls/pdf/colon.pdf $>$. Accessed 6 Feb 2012.

34. Greenland S. Introduction to stratified analysis. 2nd ed. Philadelphia: Lippincott; 1998.

35. Kurth T, et al. Results of multivariable logistic regression, propensity matching, propensity adjustment, and propensity-based weighting under conditions of nonuniform effect. Am J Epidemiol. 2006;3:262-70.

36. de Gramont A, et al. Leucovorin and fluorouracil with or without oxaliplatin as first-line treatment in advanced colorectal cancer. J Clin Oncol. 2000;16:2938-47.

37. Giacchetti S, et al. Phase III multicenter randomized trial of oxaliplatin added to chronomodulated fluorouracil-leucovorin as first-line treatment of metastatic colorectal cancer. J Clin Oncol. 2000;1:136-47.

38. Cassidy J, et al. XELOX (capecitabine plus oxaliplatin): active first-line therapy for patients with metastatic colorectal cancer. J Clin Oncol. 2004;11:2084-91.

39. Moertel CG, et al. Fluorouracil plus levamisole as effective adjuvant therapy after resection of stage III colon carcinoma: a final report. Ann Intern Med. 1995;5:321-6.

40. O'Connell MJ, et al. Controlled trial of fluorouracil and low-dose leucovorin given for 6 months as postoperative adjuvant therapy for colon cancer. J Clin Oncol. 1997;1:246-50.

41. Investigators, I.M.P.A.O.C.C.T.I. Efficacy of adjuvant fluorouracil and folinic acid in colon cancer. International Multicentre Pooled Analysis of Colon Cancer Trials (IMPACT) investigators. Lancet. $1995 ; 8955$.

42. Wolmark N, et al. The benefit of leucovorin-modulated fluorouracil as postoperative adjuvant therapy for primary colon cancer: results from National Surgical Adjuvant Breast and Bowel Project protocol C-03. J Clin Oncol. 1993;10:1879-87.

43. Grothey A, et al. Survival of patients with advanced colorectal cancer improves with the availability of fluorouracil-leucovorin, irinotecan, and oxaliplatin in the course of treatment. J Clin Oncol. 2004;7:1209-14.

44. Cartwright TH. Treatment decisions after diagnosis of metastatic colorectal cancer. Clin Colorectal Cancer. 2012;3:155-66.

45. Twelves CJ, et al. Capecitabine/oxaliplatin, a safe and active first-line regimen for older patients with metastatic colorectal cancer: post hoc analysis of a large phase II study. Clin Colorectal Cancer. 2005;2:101-7.

46. Hoff PM, et al. Comparison of oral capecitabine versus intravenous fluorouracil plus leucovorin as first-line treatment in 605 patients with metastatic colorectal cancer: results of a randomized phase III study. J Clin Oncol. 2001;8:2282-92.

47. Cassidy J, et al. Randomized phase III study of capecitabine plus oxaliplatin compared with fluorouracil/folinic acid plus oxaliplatin as first-line therapy for metastatic colorectal cancer. J Clin Oncol. 2008;12:2006-12.

48. Haller DG, et al. Potential regional differences for the tolerability profiles of fluoropyrimidines. J Clin Oncol. 2008;13:2118-23.

49. Rothenberg ML. Tolerability of fluoropyrimidines in combination with oxaliplatin appears to differ by region. In ASCO: 2008 Gastrointestinal Cancers Symposium. 2008.

50. Hochster HS, et al. Safety and efficacy of oxaliplatin and fluoropyrimidine regimens with or without bevacizumab as first-line treatment of metastatic colorectal cancer: results of the TREE Study. J Clin Oncol. 2008;21:3523-9.

51. Schrag D, et al. Who gets adjuvant treatment for stage II and III rectal cancer? Insight from surveillance, epidemiology, and end results-Medicare. J Clin Oncol. 2001;17:3712-8.

52. Hershman D, et al. Timing of adjuvant chemotherapy initiation after surgery for stage III colon cancer. Cancer. 2006;11:2581-8.

53. Dobie SA, et al. Completion of therapy by Medicare patients with stage III colon cancer. J Natl Cancer Inst. 2006;9:610-9.

54. Lund JL, et al. Identifying specific chemotherapeutic agents in medicare data: a validation study. Med Care. 2012, in press.

55. Merrill RM, et al. Survival and treatment for colorectal cancer Medicare patients in two group/staff health maintenance organizations and the fee-for-service setting. Med Care Res Rev. 1999;2:177-96.

56. Kirsner RS, et al. The effect of medicare health care delivery systems on survival for patients with breast and colorectal cancer. Cancer Epidemiol Biomark Prev. 2006;4:769-73. 